\title{
Testing if Healthy Perfectionism Enhances Academic Achievement in Australian Secondary School Students
}

\author{
Elizabeth Thorpe ${ }^{1} \&$ Ted Nettelbeck ${ }^{1}$ \\ ${ }^{1}$ School of Psychology, University of Adelaide, South Australia, Australia \\ Correspondence: Ted Nettelbeck, School of Psychology, University of Adelaide, Adelaide, South Australia 5005, \\ Australia. E-mail: ted.nettelbeck@adelaide.edu.au
}

Received: March 3, 2014

Accepted: May 20, $2014 \quad$ Online Published: June 13, 2014

doi:10.5539/jedp.v4n2p1

URL: http://dx.doi.org/10.5539/jedp.v4n2p1

\begin{abstract}
Although considerable evidence has confirmed that measures of intelligence and conscientiousness substantially predict academic achievement, other personality variables have attracted only limited research. The purpose of this study was to test the extent to which intelligence and personality variables, including perfectionism, accounted for academic grades. Participants were 180 (65 males) secondary school students in years 11-12. They completed tests for fluid and crystallised abilities (Gf, Gc), Conscientiousness (C), Openness to Experience (O), Neuroticism (N), Need for Cognition (NFC) and the Frost Multidimensional Perfectionism Scale, which was used to define healthy perfectionism (HP) and unhealthy perfectionism (UHP). Gender differences for all measures were negligible and not considered further. One aspect of HP (personal standards) overlapped moderately with NFC but HP and NFC appeared to be different constructs. Hierarchical regression found that Gf, Gc and $\mathrm{C}$ together accounted for $27 \%$ of variance in academic grade, with HP explaining an additional $6 \%$. Further contribution from NFC was not statistically significant. N correlated with UHP but did not impact grade. Higher concern about parental criticisms correlated $(r=-.27)$ with lower academic grade.
\end{abstract}

Keywords: academic grade, healthy perfectionism, secondary students, unhealthy perfectionism.

\section{Introduction}

Although considerable evidence has confirmed that measures of intelligence substantially predict academic achievement (Poropat, 2009), it has long been recognised that motivational investment is also important to success (Cattell, 1943) and a number of motivational personality traits have been identified as having incremental predictive validity for academic achievement, particularly Conscientiousness (C) from the Five Factor Model (FFM) of personality (Costa \& McCrae, 1992) and, to a lesser extent, Openness to Experience (O) (Poropat, 2009; Richardson \& Abraham, 2009). Another construct advanced as improving understanding of individual differences in academic achievement is Need for Cognition (NFC), conceptualised as a bipolar continuum, with higher scores denoting higher motivation to engage in challenging, intellectual thinking (Cacioppo \& Petty, 1982). NFC has typically been found to correlate with higher grade point average (Elias \& Loomis, 2002) and, although there has been debate about whether it correlates significantly with intelligence, the sole meta-analysis yet available has suggested that any such correlation is due more to crystallised knowledge (Gc) rather than fluid ability (Gf) (Cacioppo, Petty, Feinstein \& Jarvis, 1996). However, NFC has consistently been found to correlate with C and with O (Sadowski \& Cogburn, 1997; Tuten \& Bosnjak, 2001; Woo, Harms \& Kuncel, 2007). There is therefore a question about the extent to which correlation between NFC and academic success reflects overlap between NFC and measures of intelligence and personality. Results with respect to a relationship between Neuroticism (N) and NFC have been mixed, some finding a significant negative relationship (Sadowski \& Cogburn, 1997) but others reporting no relationship (Tuten \& Bonsjak, 2001; Woo et al., 2007).

Initially, perfectionism was generally linked to maladjustment and described as the compulsive pursuit of impossible goals, although Hamachek (1978) had early proposed that perfectionism could include both healthy elements (pleasure from challenging activities) and unhealthy elements (dissatisfaction because accomplishments are never good enough). The Frost Multidimensional Perfectionism Scale (FMPS; Frost, Martin, Lahart \& Rosenblate, 1990) has been widely used to measure perfectionism in both healthy and clinical 
samples (Shafran \& Mansell, 2001). Initially, Frost et al. represented perfectionism by six related but relatively independent subscales (Personal Standards (PS), Organisation (Org), Parental Expectations (PE), Parental Criticism (PC), Concerns over Mistakes (CM), Doubts about Actions (D)), all items from which were summed to provide a total perfectionism score. However, subsequent research has established considerable consensus that the FMPS is better used to distinguish healthy perfectionism (HP; tapped by PS in combination with Org) from unhealthy perfectionism (UHP; CM, D, PE, PC combined or sub-combinations of these) (Hawkins, Watt, \& Sinclair, 2006; Hanchon, 2010; Kornblum \& Ainley, 2005; Stumpf \& Parker, 2000). Only a few studies have considered whether perfectionism measured with the FMPS contributes to academic achievement, with inconsistency among them (Bieling, Israeli, Smith \& Anthony, 2003; Rice \& Dellwo, 2002). There is, however, evidence that higher HP, measured using an alternative scale, is associated with higher grade point average (Hanchon, 2011; Rice \& Slaney, 2002).

Consideration of the items that constitute the FMPS and NFC suggests considerable overlap between the content of these scales. This impression is particularly marked for the seven FMPS items included to measure PS, an aspect of HP. Moreover, as has been the case for NFC, those aspects of the FMPS associated with HP have consistently been found to correlate with $\mathrm{C}$, whereas those aspects reflecting UHP have correlated with $\mathrm{N}$ (Stoeber, Otto \& Dalbert, 2009; Stumpf \& Parker, 2000). Additionally, mirroring correlation between O and NFC, Rice, Ashby \& Slaney (2007) reported that higher O was associated with HP.

Given that both NFC and HP have been defined as experiencing enjoyment in challenging activities (Cacioppo \& Petty, 1982; Hamachek, 1978) it is not surprising that scales for measuring these constructs would include similar content. However, such overlap if confirmed does raise important theoretical and practical questions; do HP and NFC both independently contribute to academic success? And does one instrument have advantages over the other when endeavouring better to predict academic success?

To summarise, perfectionism and NFC have attracted extensive independent bodies of research but consideration of NFC and FMPS items does suggest some overlap in the content of these scales and there are similarities in how these have been found to correlate with the personality domains $\mathrm{C}, \mathrm{O}$ and $\mathrm{N}$. These similarities therefore provided grounds for exploring overlap between HP as measured by the FMPS and NFC in relation to academic success. This study therefore aimed to test the incremental validity of both HP and NFC when predicting academic achievement but after first controlling for intelligence and personality constructs already well-established as predicting academic success. Additionally, we explored the impact of UHP on academic grade.

\section{Method}

\subsection{Participants}

Volunteers were 196 secondary school students (65 males) aged 15-18 years; $M=16.6, S D=0.72$ ), recruited from years $11(\mathrm{n}=84)$ and $12(\mathrm{n}=112)$ at two independent schools in metropolitan Adelaide. This was a sample of convenience, rather than a random sample. All agreed beforehand to provide grades from their previous term reports. Twenty four students reported that English was not their first language but whether English was the first language or not correlated only .09 $(p>.05)$ with NFC and this was accepted as evidence that English skills for all participants were equal to completing the on-line survey.

Data from 16 participants were lost because of unreliable on-line returns, leaving 180 participants (65 males). There were no grounds to suppose that students not completing the on-line survey differed from those who did in any way likely to distort the results obtained.

\subsection{Measures}

Demographic data collected at the beginning of the survey were gender, age, year level, school name, English as first or second language.

\subsubsection{Frost Multidimensional Perfectionism Scale (FMPS)}

The FMPS (Frost et al., 1990) has 35 items designed to measure six subcomponents of perfectionism [Personal Standards (PS; 7 items), Organisation (Org; 6 items), Parental Expectations (PE; 5 items), Parental Criticism (PC; 4 items), Concerns over Mistakes (CM; 9 items), Doubts about Actions (D; 4 items)]. Agreement with each statement is rated on a 4-point Likert scale $(1=$ strongly disagree through $4=$ strongly agree $)$, with higher scores suggesting more perfectionism. Seven items were adapted from past to present tense to reflect current experience. 
FMPS has high reported internal consistency reliabilities for subscales (.77 to .93; Frost et al., 1990) and good construct validity (Lynd-Stevenson \& Hearne, 1999).

\subsubsection{Need for Cognition Scale (NFC)}

Cacioppo and Petty (1982) developed the NFC scale to measure individual engagement in effortful cognitive thought. Responses to 34 items are rated on a 4-point Likert scale $(1=$ Not at all through $4=$ Very $m u c h)$. Higher scores register higher need for cognition. Typical internal consistency has been reported to be around .85 , with good validity indicated by correlations with measures of social anxiety, intrinsic motivation and GPA (Cacioppo et al., 1996).

\subsubsection{The Quick Scales}

Developed by Brebner (2003), the Quick Scales provide six items for measuring (4-point Likert scale ;1 = Not at all through $4=$ Extremely) each of the FFM personality constructs (Costa \& McCrae, 1992). Here, we used items for only Conscientiousness, Openness to Experience and Neuroticism, because of past evidence that correlations with FMPS and NFC were limited to these domains (e.g., Stumpf \& Parker, 2000; Woo et al., 2007). Brebner reported acceptable internal consistency (.77 to .82$)$ and test-retest reliability (.65 to .86$)$, and good validity as indicated by concurrent correlation with facets of the NEO Personality Inventory-Revised (Costa \& McCrae, 1992), for these domains.

\subsubsection{Intelligence}

Ten items from the Spatial Reasoning subtest and 25 from the Vocabulary subtest of the Multidimensional Aptitude Battery - II (Jackson, 2003) were selected following pilot testing to minimise the length of the full test battery while avoiding floor and ceiling effects. Jackson reported a range in Cronbach's alpha of .94 to .95 and .72 to .76 for Spatial Reasoning and Vocabulary, respectively, among 16-17 year olds on the full test. Spatial Reasoning and Vocabulary served as markers for Gf and Gc, respectively.

\subsubsection{Academic Achievement}

The mean of students' grades (A-D) for each subject in their report from the previous term was coded 4-1, so that higher scores reflected higher grades.

\subsection{Procedure}

All measures were presented in their original format, including instructions, but online using Survey Monkey. This program provided secure access to the survey by a hyper link sent to a designated email address and permitted convenient data download for statistical analysis. Informed consent was provided in submission of the survey. Completion of the questionnaire took between 15-30 min.

\section{Results}

Sample sizes for statistical analyses ranged from 136 to 180 because some participants did not complete all measures.

\subsection{Reliability of Measures}

Cronbach's alpha coefficients for all measures were satisfactory and consistent with previously published results; FMPS .60 to .91 for the six perfectionism subscales; NFC .88; Quick Scales .62 to .76; Spatial Reasoning .84; Vocabulary .77. Vocabulary correlated .31 with Spatial Reasoning, consistent with relative independence.

\subsection{Descriptive Statistics}

Descriptive statistics are set out in Table 1. No outliers were evident for any of the tests. Means and SDs for the FMPS subscales considered in previous research to represent UHP (CM, D, PE, PC) were equivalent to previously published results (Hawkins et al., 2000; Kornblum \& Ainley, 2005; Stumpf \& Parker, 2000) but subscales representing HP (PS \& Org) were higher than average. Participants' academic grades and scores on $\mathrm{NFC}, \mathrm{N}, \mathrm{O}$ and $\mathrm{C}$ were higher than average but consistent with expectations for students in final years of secondary education at private schools that promote academic achievement. 
Table 1. Means (M), Standard Deviations (SDs) and possible ranges for all variables

\begin{tabular}{lllll}
\hline Variable & $N$ & $M(S D)$ & Minimum & Maximum \\
\hline Grade & 180 & $3.15(0.74)$ & 1 & 4 \\
FMPS subtests & & & & \\
CM & 180 & $19.66(6.25)$ & 9 & 36 \\
PS & 180 & $19.87(4.42)$ & 8 & 28 \\
D & 180 & $10.20(2.63)$ & 4 & 16 \\
Org & 180 & $17.91(4.34)$ & 6 & 24 \\
PE & 180 & $12.68(3.48)$ & 5 & 16 \\
PC & 180 & $8.14(3.08)$ & 4 & 127 \\
NFC & 158 & $93.17(14.40)$ & 52 & 24 \\
Personality & & & & 24 \\
N & 152 & $16.26(3.94)$ & 7 & 24 \\
O & 152 & $18.80(2.86)$ & 10 & 10 \\
C & 152 & $18.60(3.14)$ & 9 & 25 \\
Cognitive ability & & & & \\
Gf & 136 & $6.98(2.89)$ & 0 & 10 \\
Gc & 151 & $13.15(4.41)$ & & \\
\hline
\end{tabular}

Note . Grade $=$ academic achievement; FMPS $=$ Frost Multidimensional Perfectionism Scale; $\mathrm{CM}=$ Concerns over Mistakes; PS= Personal Standards; $\mathrm{D}=$ Doubts about Actions; Org= Organisation; $\mathrm{PE}=$ Parental Expectations; $\mathrm{PC}=$ Parental Criticisms; $\mathrm{NFC}=$ Need for Cognition; $\mathrm{N}=$ Neuroticism; $\mathrm{O}=$ Openness to Experience; $\mathrm{C}=$ Conscientiousness; $\mathrm{Gf}=$ Fluid ability (spatial reasoning); $\mathrm{Gc}=$ Crystallised ability (vocabulary).

\subsection{Correlations}

The correlation matrix is presented in Table 2. As expected, correlations between academic grade and the intelligence measures and $\mathrm{C}$ were moderately strong. $\mathrm{O}$ was not correlated with grade and has not been considered further. Weak but statistically significant correlations between gender and both $\mathrm{CM}$ and Org suggested slightly higher concern over mistakes and organisation among females but otherwise there was no evidence for sex differences. N correlated negatively with NFC and correlated positively with UHP subscales D, PC and CM (but not PE) but did not correlate with academic grade. HP variables PS and Org were moderately positively correlated with academic grade and PC (subscale of UHP) was moderately negatively correlated with grade. NFC was positively correlated with PS (subscale of HP), suggesting some overlap between the constructs, and also correlated positively with academic grade, Gf and Gc. 
Table 2. Pearson correlations for all variables

\begin{tabular}{|c|c|c|c|c|c|c|c|c|c|c|c|c|c|c|}
\hline & Gender & Grade & CM & PS & D & Org & PE & PC & NFC & $\mathbf{N}$ & $\mathbf{O}$ & C & Gf & Ge \\
\hline Gender & 1 & & & & & & & & & & & & & \\
\hline Grade & .02 & 1 & & & & & & & & & & & & \\
\hline CM & $.18^{*}$ & .15 & 1 & & & & & & & & & & & \\
\hline PS & .07 & $.37 * *$ & $.55^{* *}$ & 1 & & & & & & & & & & \\
\hline D & .11 & -.13 & $.54 * *$ & $.27 * *$ & 1 & & & & & & & & & \\
\hline Org & $.18^{*}$ & $.29 * *$ & $.20^{*}$ & $.45 * *$ & .08 & 1 & & & & & & & & \\
\hline $\mathrm{PE}$ & -.10 & -.10 & $.30^{* *}$ & .04 & $.23^{* *}$ & -.15 & 1 & & & & & & & \\
\hline $\mathrm{PC}$ & -.10 & $-.27 * *$ & $.34 * *$ & -.09 & $.39 * *$ & $-.27^{* *}$ & $.68 * *$ & 1 & & & & & & \\
\hline NFC & -.07 & $.31 * *$ & .09 & $.42 * *$ & -.04 & .05 & -.05 & -.15 & 1 & & & & & \\
\hline $\mathrm{N}$ & .15 & -.10 & $.37 * *$ & .03 & $.50^{* *}$ & -.07 & .08 & $.30^{* *}$ & $-.26^{* *}$ & 1 & & & & \\
\hline $\mathrm{O}$ & .02 & -.03 & .03 & .09 & .04 & -.13 & -.00 & .02 & $.26 * *$ & .02 & 1 & & & \\
\hline C & .11 & $.33 * *$ & $.18^{*}$ & $.59 * *$ & .03 & $.61 * *$ & -.12 & $-.26^{* *}$ & $.33 * *$ & $-.20^{*}$ & -.04 & 1 & & \\
\hline Gf & -.08 & $.22^{*}$ & $.19^{*}$ & $.25 * *$ & .08 & -.02 & .17 & .14 & $.18^{*}$ & .02 & $.19 *$ & .09 & 1 & \\
\hline $\mathrm{Gc}$ & -.09 & $.37 * *$ & .05 & .08 & -.02 & -.12 & .02 & -.02 & $.20^{*}$ & -.04 & -.04 & -.06 & $.31 * *$ & 1 \\
\hline
\end{tabular}

Note. $N=136$ (cases deleted list-wise). Gender $($ male $=1$; female $=2)$; Grade $=$ academic achievement; $\mathrm{CM}=$ Concerns over Mistakes; PS= Personal Standards; D = Doubts about Actions; Org= Organisation; PE = Parental Expectations; $\mathrm{PC}=$ Parental Criticisms; $\mathrm{NFC}=$ Need for Cognition; $\mathrm{N}=$ Neuroticism; $\mathrm{O}=$ Openness to Experience; $\mathrm{C}=$ Conscientiousness; $\mathrm{Gf}=$ Fluid ability (spatial reasoning); $\mathrm{Gc}=$ Crystallised ability (vocabulary).

$* p<.05, * * p<.01$.

\subsection{Factor Analyses}

Following Stumpf and Parker (2000), we initially checked that a 4-factor solution provides a parsimonious description for the FMPS. The 35 items were first subjected to principal components analysis. Bartlett's test of sphericity was statistically significant $\left(\chi^{2}(595)=3579.41, p<.001\right)$, indicating that correlations were sufficiently large for factorability and the Kaiser-Meyer-Olkin (KMO) measure was .87 , confirming that the sample was adequate for analysis. Inspection of the scree plot together with parallel roots analysis confirmed four factors as providing the most parsimonious solution. Factor 1 combined subscales $\mathrm{CM}$ and $\mathrm{D}$ into CMD, factor 2 combined PE and PC into PEC; factor 3 represented subscale PS; and factor 4 represented subscale Org, with $55.5 \%$ total variance among items explained. Next, a principal axis factor rotation (promax) was performed. As anticipated, all FMPS items loaded neatly and strongly on their corresponding factor except for Item 18 ("I hate being less than the best at things"), which has previously been reported as problematic (Hawkins et al., 2006; Kornblum \& Ainley, 2005). In this model, items were grouped into their associated factors in the same way as for the principal components solution. This model similarly explained $50.2 \%$ of test variance, with components representing CMD, Org, PEC and PS explaining 22.7\%, 16.9\%, 6.1\% and 4.5\%, respectively. This model distinguished between HP and UHP, with all factors showing strong internal consistency. Moreover, moderate intercorrelations between HP components PS and Org (.46) and between UHP components CMD and PEC (.42) were consistent with the suggestion that two higher-order factors representing HP and UHP more clearly describe the structure of the FMPS.

To test this, a second-order factor analysis was carried out on the first-order factor correlation matrix. The KMO was .49 (considered mediocre rather than good; Field, 2009) but Bartlett's test of Sphericity was statistically significant, indicating that correlations were sufficiently large for factorability $\left(\chi^{2}(595)=3579.41, p<.001\right)$. Two components with eigenvalues $>1$ explained $75.5 \%$ of the variance (39.4\% and $36.2 \%$, respectively). A rotated solution (promax) explained $51 \%$ of the total variance and, as suggested by previous research (Stumpf \& Parker, 2000), first-order factors PS+Org represented HP and CMD+PEC represented UHP. These second-order factors were orthogonal $(r=.05)$, suggesting that a possible third-order general factor representing overall perfectionism was unlikely. Finally, a Schmid Leiman solution was applied to the first-order and second-order 
factor solutions, allowing examination of the independent influence of first-order and higher-order factors on the 35 items of the FMPS. This analysis confirmed that the second-order factors, interpreted as HP and UHP, neatly explained the exact same amounts of variance among items and in total explained $54.4 \%$ of the variance.

\subsection{Hierarchical Multiple Regression Analyses Involving HP Variables}

Hierarchical multiple regression assessed the extent to which HP and NFC predicted academic grade after controlling for Gf, Gc and C. Gf, Gc and C were entered at Step1, with HP variables PS and Org entered at Step 2 , followed by NFC at Step 3. Step 1 accounted for $27 \%$ of variance in grade $(F(3,132)=16.09, p<.001)$. Adding HP at Step 2 accounted for an additional $6 \%(F(2,130)=5.45, p<.01)$ but adding NFC at Step 3 made little change, this analysis accounting for $33 \%$ of variance in grade. In terms of overall relative importance, only Gc and PS contributed statistically significantly to the final model $(\beta$ values $.34, p<.001 ; .20, p<.05$, respectively).

Significant correlation between NFC and PS $(.42, p<.001)$ justified rerunning this analysis with NFC entered before the HP variables. Entering NFC at Step 2 improved prediction of grade by only $1.3 \%(p>.05)$ but the HP variables at Step3 accounted for an additional $5 \%(p<.01)$, confirming the result from the first analysis.

\subsection{Correlations between UHP Variables and Academic Grade}

Correlating factor scores for the UHP variables PEC and CMD with academic grade did not improve interpretation of the correlations between grade and subtests PE, PC, CM and D. The correlation between CMD and grade was weakly positive $(r=.10)$ but not statistically significant. The correlation between PEC and grade was weakly negative $(r=-.18 ; p<.05)$ but the latter result was strongly influenced by the stronger correlation between PC and grade $(r=-.27, p<.01$; see Table 2$)$.

\section{Discussion}

The main aim was to test whether HP and NFC incrementally predicted academic achievement, beyond variance explained by intelligence together with motivational aspects of personality. This required first confirming that FMPS performance was well described by four factors (PS Org, PEC (a combination of PE and PC), and CMD (a combination of CM and D)); and that the first two and the last two defined, respectively, HP and UHP. This requirement was well met; FMPS performance was parsimoniously described in terms of these four first-order factors, consistent with studies involving German (Stöber, 1998), American (Stumpf \& Parker, 2000) and Australian samples (Harvey, Pallant \& Harvey, 2004; Hawkins et al., 2006; Kornblum \& Ainley, 2005), together with two higher-order factors, HP (PS + Org) and UHP (CMD + PEC), as proposed by Stumpf and Parker (2000). Current results found no evidence for a general perfectionism factor, confirming doubts about the validity of summing subscales to achieve a full-scale perfectionism score as advocated by Frost et al. (1990). There were, however, grounds for caution about accepting unreservedly the solution favoured here insofar as, despite low correlation between HP and UHP in the higher order analysis, the Schmid Leiman solution revealed that some items did not adequately distinguish between HP and UHP. Thus, some items measuring PEC loaded positively and strongly on UHP, as expected, but also strongly and negatively on HP, suggesting that these items could be better interpreted as reflecting a unidimensional continuum for perfectionism. Similarly, some CMD items loaded positively on both HP and UHP. This may explain why CMD, which contributed to UHP, correlated positively, albeit non-significantly, with academic grade. However, the seven items measuring the first-order HP factor PS and the six items for the first-order factor Org were all found to have strong positive loadings on the HP factor, with only small loadings on the UHP factor. In short, we are more confident about results here involving HP than those involving UHP.

Hierarchical multiple regression found that, after controlling for Gf, Gc and C, HP scores accounted for an additional $6 \%$ variance in academic grade, with higher HP related to higher academic achievement. This is an important finding; as would be expected from past research, academic grade was clearly aligned with intelligence, particularly $\mathrm{Gc}$, and $\mathrm{C}$ but in subsequent stages of regression modelling the most important markers to academic success were, first, Gc, followed by PS. The relative importance of C and of NFC have been suppressed in this sample by moderately strong correlations between PS, C and NFC and all predicted academic grade to about the same extent. Future research could consider possible overlap between $\mathrm{C}$ and HP. Despite some overlap, however, NFC and HP appear to be different constructs. Thus, NFC did not correlate with Org, the second component of HP; and NFC did not improve prediction of grade, whether entered into the regression model ahead of the HP variables or following, whereas the HP variables accounted for an additional $5-6 \%$ of variance in academic grade. Nevertheless, although HP as defined here derived from only 13 items, compared 
with 34 items in the NFC scale, it would be premature to conclude that HP provides a "better" motivational variable than NFC for predicting academic success. NFC purports to register wider intellectual curiosity, rather than a focus on excelling within more narrow areas of an educational curriculum and it may therefore be important to include both considerations in academic settings different to that engaged here. The current Australian educational climate tends to emphasise academic achievement as the door keeper to tertiary studies and, noting that our sample was not randomly selected, together with the higher than average HP scores in our sample, it is possible that many of these students, drawn from the fee-based private educational sector, were more focused on competitive markers as an indication of success than on broadening their knowledge (Harackiewicz, Barron \& Elliot, 1998). At this stage of knowledge, therefore future research should continue to include markers for the potentially separable constructs C, NFC and HP and should extend to students recruited from a wider range of socioeconomic backgrounds. Moreover, it will be important to establish if the salience of $\mathrm{HP}$ as an important predictor of academic achievement is realised in the context of a wider range of cognitive abilities, beyond the tests of abstract reasoning and word knowledge included here.

Of the UHP variables, only PC correlated statistically significantly with academic grade; an adverse relationship that accounted for about $7 \%$ of variance in grade. Of course, this does not mean that concern about parental criticism is responsible for lower academic grade; an obvious weakness of the current study is that the "outcome" measure grade was actually determined some months before the "predictor" measurements were made. Although there are sound theoretical grounds for proposing that predictor variables used here in the regression models do predict academic success, direction of causality implied by these models can only be tested within a longitudinal design.

However, these results have suggested two plausible hypotheses for future longitudinal research. The first is that, in addition to higher intelligence and more conscientious application, higher expectations for academic achievement, together with higher organisational standards, help to deliver higher academic grades. The second is that, if a student experiences high levels of concern about parental criticisms, this can negatively impact academic achievement. Moreover, despite some inconsistencies noted above, current evidence favours a description of HP and UHP as two separate factors, rather than two extremes of a continuum. Should this be confirmed by future research it means that it would be possible for an individual who was high in HP to simultaneously be high in UHP, in which case any gains from the former could be negated by the latter. Clearly, therefore, further research should be directed towards improving understanding of the development of HP but particularly UHP. Ultimately one can anticipate that this should lead to interventions to improve academic performance and wellbeing of students by increasing HP but decreasing UHP.

\section{Acknowledgements}

We are grateful to the students, their parents and teachers at the schools involved for their participation.

\section{References}

Bieling, P. J., Israeli, A., Smith, J., \& Antony, M. M. (2003). Making the grade: The behavioural consequences of perfectionism in the classroom. Personality and Individual Differences, 35, 163-178. http://dx.doi.org/10.1016/S0191-8869(02)00173-3 .

Brebner, J. (2003). The Quick Scales-R. Adelaide, South Australia: Psychology Department, University of Adelaide.

Cacioppo, J. T., \& Petty, R. E. (1982). The need for cognition. Journal of Personality and Social Psychology, 42, 116-131. http://dx.doi.org/10.1037/0022-3514.42.1.116

Cacioppo, J. T., Petty, R. E., Feinstein, J. A., \& Jarvis, B. G. (1996). Dispositional differences in cognitive motivation: The life and times of individuals varying in need for cognition. Psychological Bulletin, 119, 197-253. http://dx.doi.org/10.1037/0033-2909.119.2.197

Cattell, R. B. (1943). The description of personality: I. Foundation of trait measurement. Psychological Review, 50, 559-594. http://dx.doi.org/10.1037/h0057276

Costa, P. T., \& McCrae, R. R. (1992). Revised NEO Personality Inventory (NEO-PI-R) and NEO Five-Factor Inventory (NEO-FFI) manual. Odessa, FL: Psychological Assessment Resources.

Elias, S. M., \& Loomis, R. J. (2002). Utilizing need for cognition and perceived self efficacy to predict academic performance. Journal of Applied Social Psychology, 32, 1687-1702. http://dx.doi.org/10.1111/j.1559-1816.2002.tb02770.x 
Field, A. P. (2009). Discovering Statistics using SPSS (3rd ed.). Los Angeles: SAGE.

Frost, R. O., Martin, P., Lahart, C., \& Rosenblate, R. (1990). The dimensions of perfectionism. Cognitive Therapy and Research, 14, 449-468. http://dx.doi.org/10.1007/BF01172967

Hamachek, D. (1978). Psychodynamics of normal and neurotic perfectionism. Psychology, 15, 27-33.

Hanchon, T. A. (2010). The relations between perfectionism and achievement goals. Personality and Individual Differences, 49, 855-890. http://dx.doi.org/10.1016/j.paid.2010.07.023

Hanchon, T. A. (2011). Examining perfectionism through the lens of achievement goal theory. North American Journal of Psychology, 12, 469-490.

Harackiewicz, J. M., Barron, K. E., \& Elliot, A. J. (1998). Rethinking achievement goals: When are they adaptive for college students and why? Educational Psychologist, 33, 1-21. http://dx.doi.org/10.1207/s15326985ep3301_1

Harvey, B., Pallant, J., \& Harvey, D. (2004). An evaluation of the factor structure of the Frost Multidimensional Perfectionism Scale. Educational and Psychological Measurement, 64, 1007-1018. http://dx.doi.org/10.1177/0013164404264842

Hawkins, C. C., Watt, H. M. G., \& Sinclair, K. E. (2006). Psychometric properties of the Frost Multidimensional Perfectionism Scale with Australian adolescent girls - Clarification of the multidimensionality and perfectionist typology. Educational and Psychological Measurement, 66, 1001-1022. http://dx.doi.org/10.1177/0013164405285909

Jackson, D. N. (2003). Multidimensional Aptitude Battery -II: Manual. Port Huron, MI: Sigma Assessment Systems.

Kornblum, M., \& Ainley, M. (2005). Perfectionism and the gifted: A study of an Australian school sample. International Education Journal, 6, 232-239.

Lynd-Stevenson, R. M., \& Hearne, C. M. (1999). Perfectionism and depressive affect: The pros and cons of being a perfectionist. Personality and Individual Differences, 26, 549-562. http://dx.doi.org/10.1016/S0191-8869(98)00170-6

Poropat, A.E. (2009). A meta-analysis of the five-factor model of personality and academic performance Psychological Bulletin, 135, 322-338. http://dx.doi.org/10.1037/a0014996

Rice, K. G., Ashby, J. S., \& Slaney, R. B. (2007). Perfectionism and the five-factor model of personality. Assessment, 14, 385-398. http://dx.doi.org/10.1177/1073191107303217

Rice, K. G., \& Dellwo, J. P. (2002). Perfectionism and self-development: Implications for college adjustment. $\begin{array}{lllll}\text { Journal of Counseling } & \text { Development, } & \text { 80, }\end{array}$ http://dx.doi.org/10.1002/j.1556-6678.2002.tb00182.x

Rice, K. G., \& Slaney, R. B. (2002). Cluster of perfectionists: Two studies of emotional adjustment and academic achievement. Measurement and Evaluation in Counseling and Development, 35, 35-48.

Richardson, M., \& Abraham, C. (2009). Conscientiousness and achievement motivation predict performance. European Journal of Personality, 23, 589-605. http://dx.doi.org/10.1002/per.732

Sadowski, C. J., \& Cogburn, H. E. (1997). Need for cognition in the big-five factor structure. The Journal of Psychology, 131, 307-312. http://dx.doi.org/10.1080/00223989709603517

Shafran, R., \& Mansell, W. (2001). Perfectionism and psychopathology: A review of research and treatment. Clinical Psychology Review, 21, 879-906. http://dx.doi.org/10.1016/S0272-7358(00)00072-6

Stöber, J. (1998). The Frost Multidimensional Perfectionism Scale Revisited: More perfect with four (instead of six) dimensions. Personality and Individual Differences, 24, 481-491. http://dx.doi.org/10.1016/S0191-8869(97)00207-9

Stoeber, J., Otto, K., \& Dalbert, C. (2009). Perfectionism and the big five: Conscientiousness predicts longitudinal increases in self orientated perfectionism. Personality and Individual Differences, 47, 363-368. http://dx.doi.org/10.1016/j.paid.2009.04.004 
Stumpf, H., \& Parker, W. D. (2000). A hierarchical structural analysis of perfectionism and its relation to other personality characteristics. Personality and Individual Differences, 28, 837-852. http://dx.doi.org/10.1016/S0191-8869(99)00141-5

Tuten, T. L., \& Bosnjak, M. (2001). Understanding differences in web usage: The role of Need for Cognition and the Five Factor Model of personality. Social Behavior and Personality, 29, 391-398. http://dx.doi.org/10.2224/sbp.2001.29.4.391

Woo, S. E., Harms, P. D., \& Kuncel, N. R. (2007). Integrating personality and intelligence: Typical intellectual engagement and need for cognition. Personality and Individual Differences, 43, 1635-1639. http://dx.doi.org/10.1016/j.paid.2007.04.022

\section{Copyrights}

Copyright for this article is retained by the author(s), with first publication rights granted to the journal.

This is an open-access article distributed under the terms and conditions of the Creative Commons Attribution license (http://creativecommons.org/licenses/by/3.0/). 